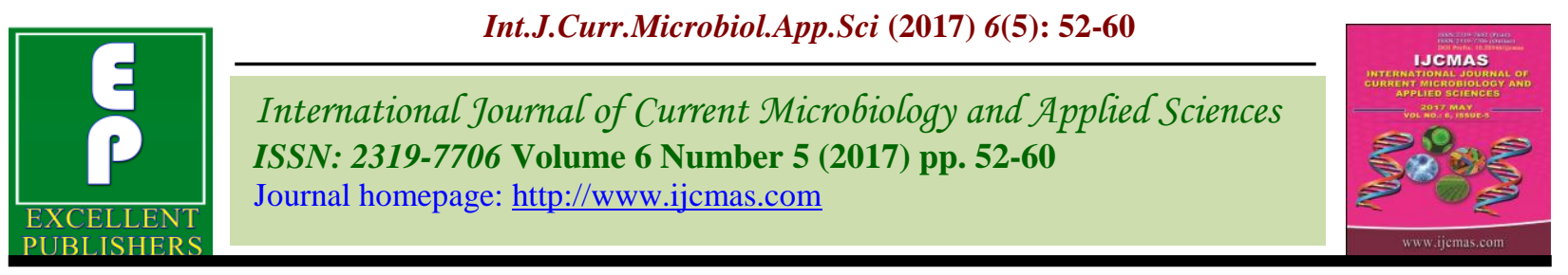

Original Research Article https://doi.org/10.20546/ijcmas.2017.605.007

\title{
Response of Land Configuration and Deficit Irrigation on Growth and Yield Attributes of Maize (Zea mays L.)
}

\author{
Hanamant M. Halli* and S.S. Angadi \\ Department of Agronomy, University of Agricultural Sciences, \\ Dharwad-580005 (Karnataka), India \\ *Corresponding author
}

\begin{tabular}{|c|c|}
\hline & A B S T R A C T \\
\hline Keywords & $\begin{array}{l}\text { The field experiment was conducted during summer } 2015 \text { and } 2016 \text { under irrigated } \\
\text { condition to study the effect of land configurations and deficit irrigation on maize at the }\end{array}$ \\
\hline $\begin{array}{l}\text { Ridges and furrow, } \\
\text { corrugated furrow, } \\
\text { Broad bed and } \\
\text { furrow, depletion of } \\
\text { available soil } \\
\text { moisture (DASM) } \\
\text { and maize. }\end{array}$ & $\begin{array}{l}\text { Main Agriculture Research Station, University of Agricultural Sciences, Dharwad, } \\
\text { Karnataka. The treatments include land configurations (Broad bed and furrow; BBF, } \\
\text { corrugated furrow and ridges and furrow methods) and irrigation levels (Irrigation once in } \\
\text { ten days, irrigation at } 40,50 \text { and } 60 \text { per cent depletion). Results revealed that, ridges and } \\
\left.\text { furrow ( } 70.6 \mathrm{q} \mathrm{ha}^{-1}\right) \text { and corrugated furrow method }\left(68.9 \mathrm{q} \mathrm{ha}^{-1}\right) \text { produced significantly } \\
\text { higher and on par grain yield as compared to BBF method of planting }\left(60.9 \mathrm{q} \mathrm{ha}^{-1}\right) \text {. } \\
\text { Whereas, irrigation at } 50 \text { per cent }\left(69.7 \mathrm{q} \mathrm{ha}^{-1}\right) \text { and } 40 \text { per cent }\left(69.6 \mathrm{q} \mathrm{ha}^{-1}\right) \text { depletion }\end{array}$ \\
\hline Article Info & \\
\hline $\begin{array}{l}\text { Accepted: } \\
\text { 04 April } 2017 \\
\text { Available Online: } \\
10 \text { May } 2017\end{array}$ & $\begin{array}{l}74.9 \mathrm{q} \mathrm{ha}^{-1} \text { ) and ridges and furrow method with irrigation at } 40 \text { (grain yield of } 72.8 \mathrm{q} \mathrm{ha}^{-1} \text { ) } \\
\text { as well as irrigation at } 50 \text { per cent depletion significantly enhanced the growth and yield } \\
\text { attributes as compared to the rest of the treatment combinations. BBF method with } \\
\text { irrigation at } 60 \text { per cent depletion recorded significantly lowest growth and yield } \\
\text { parameters of maize. }\end{array}$ \\
\hline
\end{tabular}

\section{Introduction}

Maize (Zea mays L.) is the third most important diversified and high potential cereal crop (after wheat and rice) and is grown throughout a wide range of climates. It is desired for multiple purposes as human food, animal feed, pharmaceutical, industrial manufacturing, corn syrup and oil, hence it is gradually replacing other crops too. The major producers are USA followed by China, Brazil, Argentina and India. In India it occupied an area of $9.5 \mathrm{~m}$ ha with production of $25 \mathrm{~m} \mathrm{t}$ and the productivity of $2500 \mathrm{~kg} \mathrm{ha}^{-1}$, which is less than half of the world productivity.
In India maize accounts for 10 per cent of the total food grain production, its area and production are increasing gradually in both irrigated and rainfed area because of multiple demands and better market price (Anon., 2015a).

The productivity of any crop is the complex phenomenon and governed by number of factors viz., use of improved genotypes, appropriate time and method of sowing and judicious use of water as well as nutrients and other management practices. However, appropriate agronomic management practices 
like suitable land configuration and regulated water usage are the most critical factors for realizing desired yield potential with higher resource use efficiency (Deshmukh et al., 2016).

Land configurations is important for better growth and development of any crop and decides the effectiveness of the crop management practices, regarding application of nutrient, irrigation water, weed management, etc. They have an impact on the crop growth by influencing the soil moisture availability, aeration, root growth, crop lodging and nutrient availability. Deficit irrigation practices irrigate the entire crop root zone with less amount of water than the conventional method of irrigation. The mild stress has minimal effects on the crop yield and expected to trigger different water stress mechanisms in crop. Irrigating crops with desirable depletion is not practiced which leads to excessive use of water than the crop requirement.

\section{Materials and Methods}

The field experiment was conducted during summer 2015 and 2016 at the Main Agriculture Research Station, University of Agricultural Sciences, Dharwad (Karnataka), situated at $15^{\circ} 26^{\prime} \mathrm{N}$ latitude, $75^{\circ} 07^{\prime} \mathrm{E}$ longitude and at an altitude of $678 \mathrm{~m}$ above mean sea level. The research station comes under Northern Transition Zone (Zone-8) of Karnataka. The soil type of the experimental site was medium black (vertisols) and clayey in texture. The soil was neutral to slightly alkaline in reaction (7.83) with normal in electrical conductivity $\left(0.24 \mathrm{dS} \mathrm{m} \mathrm{m}^{-1}\right)$, medium in organic carbon content $(0.62 \%)$, medium in available of nitrogen $\left(320.3 \mathrm{~kg} \mathrm{ha}^{-1}\right)$ and phosphorus (33.21 kg ha-1) and high in available potassium $\left(426.5 \mathrm{~kg} \mathrm{ha}^{-1}\right)$. The bulk density of top soil $(30 \mathrm{~cm})$ was $1.24 \mathrm{~g} \mathrm{cc}^{-1}$. The soil moisture content at field capacity was 32.40 per cent and permanent wilting point was 18.00 per cent in the upper 0 to $30 \mathrm{~cm}$ surface. The total rainfall received during maize growing period varied with respect to amount and intensity (February to May) and was $247.8 \mathrm{~mm}$ during 2015 and $105.8 \mathrm{~mm}$ during 2016 with a rainy days of 11 during 2015 and 7 days during 2016. Higher amount of rainfall was received during May month i.e., $129.4 \mathrm{~mm}$ during 2015 and $82.8 \mathrm{~mm}$ during 2016. The mean maximum temperatures recorded during crop growth period were highest in April second fortnight $\left(35.5{ }^{\circ} \mathrm{C}\right.$ during 2015 and $38.6{ }^{\circ} \mathrm{C}$ during 2016) and lowest were in February first fortnight $\left(30.9^{\circ} \mathrm{C}\right.$ during 2015 and 32.6 ${ }^{\circ} \mathrm{C}$ during 2016). The average evaporation rate was highest during $2016\left(7.65 \mathrm{~mm} \mathrm{day}^{-1}\right)$ as compared to 2015 (5.92 $\left.\mathrm{mm} \mathrm{day}^{-1}\right)$. Similarly, the average soil temperature was higher in April first fortnight $\left(44.71^{\circ} \mathrm{C}\right)$ during 2016 as compared to $42.10{ }^{\circ} \mathrm{C}$ during 2015 .

The experiment was laid out in split plot design with three replications and comprising twelve treatment combinations with three land configurations as main plot and four irrigation levels as sub plot. Land configurations include, $\mathrm{L}_{1}$ : Broad bed and furrow $(\mathrm{BBF}), \mathrm{L}_{2}$ : Corrugated (shallow) furrow and $\mathrm{L}_{3}$ : Ridges and furrow. Whereas, irrigation levels include, $\mathrm{I}_{1}$ : Irrigation once in ten days, $\mathrm{I}_{2}$ : Irrigation at 40 per cent depletion of available soil moisture (DASM), $\mathrm{I}_{3}$ : Irrigation at 50 per cent DASM and $\mathrm{I}_{4}$ : Irrigation at 60 per cent DASM. The net plot area was $3.6 \times 4.6 \mathrm{~m}$ and the hybrid grown was Pinnacle at the recommended spacing of $60 \times 20 \mathrm{~cm}$. Crop was planted on February $6^{\text {th }}$ and harvested on May $31^{\text {st }}$ in 2015 and planted on February $1^{\text {st }}$ and harvested on May $24^{\text {th }}$ in 2016. Broad bed and furrows were prepared with length of 5.4 $\mathrm{m}, 90 \mathrm{~cm}$ width and $12.5 \mathrm{~cm}$ deep furrow and each bed occupied two crop rows. With respect to corrugated furrows, shallow depth of $10 \mathrm{~cm}$ was maintained. Similarly for ridges and furrows at a depth of $25 \mathrm{~cm}$ was 
maintained. The care was taken to maintain the same number of crop rows (10 rows) and total number of plants in each configuration. The shape of the configurations was maintained through the crop duration. The space of $1 \mathrm{~m}$ was maintained between main plots and $0.4 \mathrm{~m}$ between sub plots. Similarly separate irrigation channels were prepared in between the main plots as buffer furrow to maintain the treatment effect and to avoid the entry of excess rain or irrigation water in to field. Urea, single super phosphate and muriate of potash were used as sources of NPK at recommended dosages of 150:75:37.5 $\mathrm{kg} \mathrm{N}: \mathrm{P}_{2} \mathrm{O}_{5}: \mathrm{K}_{2} \mathrm{O}$ ha $^{-1}$. Fifty per cent of nitrogen and 100 per cent phosphorus and potassium were applied as basal dose and remaining 50 per cent of $\mathrm{N}$ was applied in two splits at 30 DAS and at tasseling stage. The field was irrigated commonly with sprinkler immediately after sowing for the uniform germination and establishment of crop. Twenty days after sowing irrigation was scheduled according to the per cent depletion of available soil moisture. Prior to each irrigation soil moisture content was measured by using Theta probe.

The quantity of water discharged was measured by Parshall flume (Michael, 2009). The per cent depletion of available soil moisture was calculated by using the following formula and also time required to irrigate each configuration was recorded. The depth of water supplied through irrigation and rainfall was accounted to total depth of water applied treatment wise.

Per cent depletion of ASM =

$$
\frac{\text { FC-PWP) } x \% \text { depletion }}{100}
$$

Where, FC- field capacity, PWP- permanent wilting point, ASM- available soil moisture.

\section{Results and Discussion}

The two years (pooled) data revealed that growth and yield parameters of maize differed significantly due to land configurations, irrigation levels and their interaction effects.

\section{Growth parameters}

Among planting methods, significantly higher plant height was recorded with ridges and furrow method $(154.2 \mathrm{~cm})$ and was superior as compared to corrugated furrow $(152.6 \mathrm{~cm})$ and broad BBF method of planting, which recorded lowest plant height $(150.1 \mathrm{~cm})$ at harvest (Table 1). Better availability of soil moisture and proper root aeration with ridges and furrow method might have favoured cell elongation and division leading to higher plant height of maize as compared to $\mathrm{BBF}$, where partial root stress led to shorter plants. These results are in conformity with the findings of Kang et al., (2000) and Fusheng et al., (2007). Similarly, irrigation at 50 per cent depletion of available soil moisture (DASM) recorded significantly higher plant height $(154.7 \mathrm{~cm})$ and was on par with irrigation at 40 per cent DASM $(154.0 \mathrm{~cm})$ and both were superior over irrigation once in ten days $(150.7 \mathrm{~cm})$ and irrigation at 60 per cent DASM $(149.9 \mathrm{~cm})$.

Higher plant height with irrigation at 50 and 40 per cent DASM might be associated with stimulated vegetative growth due to favoured soil moisture regimes. Whereas, in irrigation at 60 per cent DASM moisture stress might promote lignin synthesis, leading to increased stiffness of cell wall and reduction in cell elongation which resulted in reduced plant height. Similar results were reported by Girijesh et al., (2011) and Silungwe et al., (2010). The interaction effect due to ridges and furrow method of planting with irrigation at $50(156.4 \mathrm{~cm})$ and 40 per cent DASM $(155.4 \mathrm{~cm})$ recorded significantly higher plant 
height and were on par with corrugated furrow at $50(154.7 \mathrm{~cm})$ and 40 per cent DASM $(154.3 \mathrm{~cm})$ as compared to other treatment combinations. However, BBF method in combination with irrigation at 60 per cent DASM recorded significantly lowest plant height $(147.5 \mathrm{~cm})$.

The leaf area recorded was significantly higher with ridges and furrow method of planting $\left(3836 \mathrm{~cm}^{2}\right.$ plant $\left.{ }^{-1}\right)$ and was remained on par with corrugated furrow method (3733 $\mathrm{cm}^{2}$ plant $^{-1}$ ) as compared to BBF method $\left(3476 \mathrm{~cm}^{2}\right.$ plant $^{-1}$ ) at 90 DAS (Table 2). Taller plants in ridges and furrow might have favoured photosynthesis and assimilation of photosynthates hence higher leaf area was recorded as compared to BBF (Patil and Sheelavantar 2001 and Tumbare and Bhoite 2000). Similarly, irrigation at 40 per cent DASM recorded significantly higher leaf area (3774 $\mathrm{cm}^{2}$ plant $^{-1}$ ) and was on par with irrigation at 50 per cent DASM $\left(3730 \mathrm{~cm}^{2}\right.$ plant $\left.^{-1}\right)$ as compared to irrigation once in ten days $\left(3638 \mathrm{~cm}^{2}\right.$ plant $\left.^{-1}\right)$ and irrigation at 60 per cent DASM $\left(3584 \mathrm{~cm}^{2}\right.$ plant $\left.^{-1}\right)$.

Taller plants with irrigation at 40 per cent DASM might be due to better photosynthesis which produced higher leaf area as compared to irrigation at 60 per cent DASM (Abdullah et al., 2015 and Yazar et al., 2009). Significantly higher leaf area was recorded with ridges and furrow method with irrigation at 40 per cent DASM $\left(3876 \mathrm{~cm}^{2}\right.$ plant $\left.^{-1}\right)$. However, this treatment found statistically on par with ridges and furrow method with irrigation at 50 per cent DASM $\left(3810 \mathrm{~cm}^{2}\right.$ plant $\left.^{-1}\right)$, corrugated furrow with irrigation at 50 per cent DASM (3808 $\mathrm{cm}^{2}$ plant $\left.^{-1}\right)$, whereas, significantly lowest leaf area was produced with $\mathrm{BBF}$ method at 60 per cent DASM $\left(3238 \mathrm{~cm}^{2}\right.$ plant $\left.^{-1}\right)$.

Significantly higher canopy temperature was observed with BBF method of planting $\left(34.7^{\circ} \mathrm{C}\right)$ and was statistically superior over corrugated furrow method $\left(33.8^{\circ} \mathrm{C}\right)$ and ridges and furrow method of planting $\left(33.2^{\circ} \mathrm{C}\right.$ ) at harvest (Table 3$)$. Better moisture availability promoted plant growth and created favourable microclimate that resulted in lower canopy temperature in ridges and furrow as compared to BBF method of planting.

In general, canopy temperature followed the increasing trend with increased soil moisture stress. At harvest, irrigation at 60 per cent DASM observed significantly higher canopy temperature $\left(35.4^{\circ} \mathrm{C}\right)$, whereas, irrigation at 40 per cent DASM recorded significantly lower canopy temperature $\left(33.0^{\circ} \mathrm{C}\right)$.

Frequent irrigation at lower moisture depletion might result in better crop growth and lower canopy temperature in ridges and furrow over BBF method. The interaction effect of canopy temperature was significantly higher with BBF method of planting at 60 per cent DASM $\left(36.1^{\circ} \mathrm{C}\right)$ which was at par with corrugated furrow method at 60 per cent $\operatorname{DASM}\left(35.5^{\circ} \mathrm{C}\right)$.

Whereas, ridges and furrow method of planting at 40 per cent DASM $\left(32.4^{\circ} \mathrm{C}\right)$ recorded significantly lower canopy temperature and was on par with corrugated method at 40 per cent DASM $\left(32.7^{\circ} \mathrm{C}\right)$ as compared to rest of treatment combinations.

\section{Yield attributes}

Ridges and furrow method of planting recorded significantly higher cob weight $(185.4 \mathrm{~g})$ as compared to corrugated furrow $(178.9 \mathrm{~g})$ and BBF method of planting (163.4 g). Higher cob weight in ridges and furrow might be associated with taller plants with higher photosynthesis, translocation and accumulation of photosynthates as compared to other methods (Table 4). 
Table.1 Plant height $(\mathrm{cm})$ of maize as influenced by land configuration and irrigation levels at harvest

\begin{tabular}{|c|c|c|c|c|c|c|c|c|c|c|c|c|}
\hline \multirow{2}{*}{ I/L } & \multicolumn{4}{|c|}{2015} & \multicolumn{4}{|c|}{2016} & \multicolumn{4}{|c|}{ Pooled } \\
\hline & $\mathbf{L}_{1}$ & $\mathbf{L}_{2}$ & $\mathbf{L}_{3}$ & Mean & $\mathbf{L}_{1}$ & $\mathbf{L}_{2}$ & $\mathbf{L}_{3}$ & Mean & $\mathbf{L}_{1}$ & $\mathbf{L}_{2}$ & $\mathbf{L}_{3}$ & Mean \\
\hline $\mathbf{I}_{1}$ & $156.6^{\mathrm{ef}}$ & $159.3^{\mathrm{c}-\mathrm{f}}$ & $162.2^{b c}$ & $159.4^{\mathrm{c}}$ & $139.1^{\mathrm{c}}$ & $143.2^{\mathrm{ab}}$ & $143.7^{\mathrm{ab}}$ & $142.0^{b}$ & $147.9^{f}$ & $151.2^{\mathrm{de}}$ & $152.9^{\mathrm{b}-\mathrm{d}}$ & $150.7^{b}$ \\
\hline $\mathbf{I}_{2}$ & $159.7^{\mathrm{c}-\mathrm{e}}$ & $162.7^{b c}$ & $164.4^{\mathrm{ab}}$ & $162.3^{b}$ & $144.7^{\mathrm{ab}}$ & $145.9^{\mathrm{a}}$ & $146.5^{\mathrm{a}}$ & $145.7^{a}$ & $152.2^{\mathrm{cd}}$ & $154.3^{\mathrm{a}-\mathrm{c}}$ & $155.4^{\mathrm{a}}$ & $154.0^{\mathrm{ab}}$ \\
\hline $\mathbf{I}_{3}$ & $161.5^{b-d}$ & $164.3^{\mathrm{ab}}$ & $167.2^{\mathrm{a}}$ & $164.4^{\mathrm{a}}$ & $144.6^{\mathrm{ab}}$ & $145.1^{\mathrm{ab}}$ & $145.5^{\mathrm{ab}}$ & $145.1^{a}$ & $153.0^{b-d}$ & $154.7^{\mathrm{ab}}$ & $156.4^{\mathrm{a}}$ & $154.7^{\mathrm{a}}$ \\
\hline$I_{4}$ & $155.7^{\mathrm{f}}$ & $158.0 \mathrm{~d}-\mathrm{f}$ & $160.6^{b-d}$ & $158.1^{\mathrm{c}}$ & $139.3^{\mathrm{c}}$ & $142.2^{\mathrm{bc}}$ & $143.9^{\mathrm{ab}}$ & $141.8^{b}$ & $147.5^{\mathrm{f}}$ & $150.1^{\mathrm{e}}$ & $152.3^{\mathrm{cd}}$ & $149.9^{b}$ \\
\hline Mean & $158.4^{\mathrm{b}}$ & $161.1 a^{b}$ & $163.6^{\mathrm{a}}$ & & $141.9^{b}$ & $144.1^{\text {ab }}$ & $144.9^{\mathrm{a}}$ & & $150.1^{\mathrm{c}}$ & $152.6^{b}$ & $154.2^{\mathrm{a}}$ & \\
\hline SV & \multicolumn{4}{|c|}{ S. Em \pm} & \multicolumn{4}{|c|}{ S. Em \pm} & \multicolumn{4}{|c|}{ S. Em \pm} \\
\hline $\mathbf{L}$ & \multicolumn{4}{|c|}{0.76} & \multicolumn{4}{|c|}{0.62} & \multicolumn{4}{|c|}{0.37} \\
\hline I & \multicolumn{4}{|c|}{0.67} & \multicolumn{4}{|c|}{0.58} & \multicolumn{4}{|c|}{0.39} \\
\hline L $\times$ I & \multicolumn{4}{|c|}{1.16} & \multicolumn{4}{|c|}{1.00} & \multicolumn{4}{|c|}{0.67} \\
\hline
\end{tabular}

Table.2 Leaf area $\left(\mathrm{cm}^{2}\right.$ plant $\left.{ }^{-1}\right)$ of maize as influenced by land configuration and irrigation levels at 90 DAS

\begin{tabular}{|c|c|c|c|c|c|c|c|c|c|c|c|c|}
\hline \multirow{2}{*}{$\mathbf{I} / \mathbf{L}$} & \multicolumn{4}{|c|}{2015} & \multicolumn{4}{|c|}{2016} & \multicolumn{4}{|c|}{ Pooled } \\
\hline & $\mathbf{L}_{1}$ & $\mathbf{L}_{2}$ & $\mathbf{L}_{3}$ & Mean & $\mathbf{L}_{1}$ & $\mathbf{L}_{2}$ & $\mathbf{L}_{3}$ & Mean & $\mathbf{L}_{1}$ & $\mathbf{L}_{2}$ & $\mathbf{L}_{3}$ & Mean \\
\hline$I_{1}$ & $3484^{\mathrm{c}}$ & $3632^{\mathrm{bc}}$ & $3768^{\mathrm{ab}}$ & $3628^{\text {ab }}$ & $3412^{\mathrm{bc}}$ & $3692^{a b}$ & $3840^{\mathrm{a}}$ & $3648^{b c}$ & $3448^{\mathrm{c}}$ & $3662^{a-c}$ & $3804^{\mathrm{ab}}$ & $3638^{b c}$ \\
\hline$I_{2}$ & $3628^{b c}$ & $3748^{\mathrm{ab}}$ & $3808^{\mathrm{ab}}$ & $3728^{a}$ & $3664^{\mathrm{ab}}$ & $3852^{\mathrm{a}}$ & $3944^{\mathrm{a}}$ & $3820^{a}$ & $3646^{b c}$ & $3800^{\mathrm{ab}}$ & $3876^{\mathrm{a}}$ & $3774^{\mathrm{a}}$ \\
\hline $\mathbf{I}_{3}$ & $3456^{\mathrm{c}}$ & $3756^{\mathrm{ab}}$ & $3816^{\mathrm{ab}}$ & $3676^{\mathrm{ab}}$ & $3688^{a b}$ & $3860^{\mathrm{a}}$ & $3804^{\mathrm{a}}$ & $3784^{\text {ab }}$ & $3572^{\mathrm{c}}$ & $3808^{\mathrm{ab}}$ & $3810^{\mathrm{ab}}$ & $3730^{\text {ab }}$ \\
\hline $\mathbf{I}_{4}$ & $3136^{\mathrm{d}}$ & $3660^{b c}$ & $3952^{\mathrm{a}}$ & $3_{583}^{b}$ & $3340^{\mathrm{c}}$ & $3664^{\mathrm{ab}}$ & $3752^{\mathrm{a}}$ & $3585^{c}$ & $3238^{\mathrm{d}}$ & $3662^{a-c}$ & $3852^{\mathrm{ab}}$ & $3584^{c}$ \\
\hline Mean & $3426^{b}$ & $3699^{a}$ & $3836^{a}$ & & $3526^{b}$ & $3767^{a}$ & $3835^{a}$ & & $3476^{b}$ & 3733 $^{\mathrm{a}}$ & $3836^{a}$ & \\
\hline SV & \multicolumn{4}{|c|}{ S. Em \pm} & \multicolumn{4}{|c|}{ S. Em \pm} & \multicolumn{4}{|c|}{ S. Em \pm} \\
\hline $\mathbf{L}$ & \multicolumn{4}{|c|}{19.93} & \multicolumn{4}{|c|}{34.92} & \multicolumn{4}{|c|}{26.22} \\
\hline I & \multicolumn{4}{|c|}{43.63} & \multicolumn{4}{|c|}{52.22} & \multicolumn{4}{|c|}{38.08} \\
\hline L x I & \multicolumn{4}{|c|}{75.57} & \multicolumn{4}{|c|}{90.45} & \multicolumn{4}{|c|}{65.95} \\
\hline
\end{tabular}

\section{Land configuration $(\mathrm{L})$}

$\mathbf{L}_{\mathbf{1}}$ : Broad bed and furrow

$\mathbf{L}_{2}$ : Corrugated furrow (shallow)

$\mathbf{L}_{3}$ : Ridges and furrow
Per cent depletion of available soil moisture (I)

$\mathbf{I}_{\mathbf{1}}$ : Irrigation once in 10 days

I 2 : Irrigation at $40 \%$ depletion

$\mathbf{I}_{3}$ : Irrigation at $50 \%$ depletion

I4: Irrigation at $60 \%$ depletion 
Table.3 Canopy temperature $\left({ }^{\circ} \mathrm{C}\right)$ of maize as influenced by land configuration and irrigation levels at harvest

\begin{tabular}{|c|c|c|c|c|c|c|c|c|c|c|c|c|}
\hline \multirow{2}{*}{$\mathbf{I} / \mathbf{L}$} & \multicolumn{4}{|c|}{2015} & \multicolumn{4}{|c|}{2016} & \multicolumn{4}{|c|}{ Pooled } \\
\hline & $\mathbf{L}_{1}$ & $\mathbf{L}_{2}$ & $\mathbf{L}_{3}$ & Mean & $\mathbf{L}_{1}$ & $\mathbf{L}_{2}$ & $\mathbf{L}_{3}$ & Mean & $\mathbf{L}_{1}$ & $\mathbf{L}_{2}$ & $\mathbf{L}_{3}$ & Mean \\
\hline$I_{1}$ & $33.1^{b}$ & $31.9^{\mathrm{d}}$ & $31.2^{\mathrm{ef}}$ & $32.1^{b}$ & $37.3^{\mathrm{ab}}$ & $36.0^{\mathrm{cd}}$ & $34.9^{\text {de }}$ & $36.0^{b}$ & $35.2^{\mathrm{bc}}$ & $33.9^{\mathrm{de}}$ & $33.0^{\mathrm{fg}}$ & $34.1^{b}$ \\
\hline$I_{2}$ & $31.8^{d}$ & $30.6^{\text {gh }}$ & $30.3^{\mathrm{h}}$ & $30.9^{c}$ & $36.0^{\mathrm{cd}}$ & $34.7^{\mathrm{de}}$ & $34.5^{\mathrm{e}}$ & $35.1^{\mathrm{c}}$ & $33.9^{\mathrm{de}}$ & $32.7^{\mathrm{g}}$ & $32.4^{\mathrm{g}}$ & $33.0^{c}$ \\
\hline $\mathbf{I}_{3}$ & $31.6^{\mathrm{de}}$ & $31.1^{\mathrm{fg}}$ & $30.7^{\text {gh }}$ & $31.1^{c}$ & $35.8^{\mathrm{cd}}$ & $35.1^{\mathrm{c}-\mathrm{e}}$ & $34.7^{\text {de }}$ & $35.2^{c}$ & $33.7^{\mathrm{ef}}$ & $33.1^{\mathrm{fg}}$ & $32.7^{\mathrm{g}}$ & $33.2^{\mathrm{c}}$ \\
\hline$I_{4}$ & $34.0^{\mathrm{a}}$ & $33.6^{b}$ & $32.6^{c}$ & $33.4^{\mathrm{a}}$ & $38.2^{\mathrm{a}}$ & $37.5^{\mathrm{ab}}$ & $36.5 b^{c}$ & $37.4^{\mathrm{a}}$ & $36.1^{\mathrm{a}}$ & $35.5^{\mathrm{ab}}$ & $34.5^{\mathrm{cd}}$ & $35.4^{\mathrm{a}}$ \\
\hline Mean & $32.6^{\mathrm{a}}$ & $31.8^{b}$ & $31.2^{c}$ & & $36.8^{\mathrm{a}}$ & $35.8^{b}$ & $35.1^{\mathrm{c}}$ & & $34.7^{\mathrm{a}}$ & $33.8^{b}$ & $33.2^{c}$ & \\
\hline SV & \multicolumn{4}{|c|}{ S. Em \pm} & \multicolumn{4}{|c|}{ S. Em \pm} & \multicolumn{4}{|c|}{ S. Em \pm} \\
\hline $\mathbf{L}$ & \multicolumn{4}{|c|}{0.10} & \multicolumn{4}{|c|}{0.17} & \multicolumn{4}{|c|}{0.13} \\
\hline I & \multicolumn{4}{|c|}{0.09} & \multicolumn{4}{|c|}{0.23} & \multicolumn{4}{|c|}{0.14} \\
\hline L x I & \multicolumn{4}{|c|}{0.16} & \multicolumn{4}{|c|}{0.40} & \multicolumn{4}{|c|}{0.24} \\
\hline
\end{tabular}

*Temperature of outside air was $38.3^{\circ} \mathrm{C}(2016)$ at harvest

Table.4 Cob weight $(\mathrm{g})$ of maize as influenced by land configuration and irrigation levels

\begin{tabular}{|c|c|c|c|c|c|c|c|c|c|c|c|c|}
\hline \multirow{2}{*}{$\mathbf{I} / \mathbf{L}$} & \multicolumn{4}{|c|}{2015} & \multicolumn{4}{|c|}{2016} & \multicolumn{4}{|c|}{ Pooled } \\
\hline & $\mathbf{L}_{1}$ & $\mathbf{L}_{2}$ & $\mathbf{L}_{3}$ & Mean & $\mathbf{L}_{1}$ & $\mathbf{L}_{2}$ & $\mathbf{L}_{3}$ & Mean & $\mathbf{L}_{1}$ & $\mathbf{L}_{2}$ & $\mathbf{L}_{3}$ & Mean \\
\hline $\mathbf{I}_{1}$ & $172.7^{\mathrm{cd}}$ & $179.3^{\mathrm{ab}}$ & $185.6^{\mathrm{ab}}$ & $179.2^{\mathrm{a}}$ & $167.6^{\mathrm{cd}}$ & $174.3^{\mathrm{b}-\mathrm{d}}$ & $181.2^{\mathrm{ab}}$ & $174.4^{\mathrm{a}}$ & $170.1^{\mathrm{ef}}$ & $176.8^{\mathrm{cd}}$ & $183.4^{\mathrm{b}}$ & $176.8^{b}$ \\
\hline$I_{2}$ & $176.5^{b-d}$ & $186.2^{\mathrm{ab}}$ & $190.9^{\mathrm{a}}$ & $184.5^{\mathrm{a}}$ & $171.4^{\mathrm{b}-\mathrm{d}}$ & $181.4^{\mathrm{ab}}$ & $186.5^{\mathrm{a}}$ & $179.7^{\mathrm{a}}$ & $173.9^{\mathrm{de}}$ & $183.8^{b}$ & $188.7^{\mathrm{a}}$ & $182.1^{\mathrm{a}}$ \\
\hline $\mathbf{I}_{3}$ & $169.5^{\mathrm{d}}$ & $183.7^{\mathrm{ab}}$ & $191.9^{\mathrm{a}}$ & $181.7^{\mathrm{a}}$ & $164.4^{\mathrm{d}}$ & $179.3^{\mathrm{ab}}$ & $187.5^{\mathrm{a}}$ & $177.1^{a}$ & $167.0^{\mathrm{f}}$ & $181.5^{\mathrm{bc}}$ & $189.7^{\mathrm{a}}$ & $179.4^{\text {ab }}$ \\
\hline $\mathbf{I}_{4}$ & $145.3^{\mathrm{e}}$ & $175.6^{b-d}$ & $182 .^{1 \mathrm{a}-\mathrm{c}}$ & $167.7^{b}$ & $140.2^{\mathrm{e}}$ & $171.2^{\mathrm{b}-\mathrm{d}}$ & $177.7^{\mathrm{a}-\mathrm{c}}$ & $163.0^{b}$ & $142.7^{\mathrm{g}}$ & $173.4^{\mathrm{de}}$ & $179.9^{\mathrm{bc}}$ & $165.4^{\mathrm{c}}$ \\
\hline Mean & $166.0^{b}$ & $181.2^{\mathrm{a}}$ & $187.6^{\mathrm{a}}$ & & $160.9^{b}$ & $176.5^{\mathrm{a}}$ & $183.2^{\mathrm{a}}$ & & $163.4^{\mathrm{c}}$ & $178.9^{b}$ & $185.4^{\mathrm{a}}$ & \\
\hline SV & \multicolumn{4}{|c|}{ S. Em \pm} & \multicolumn{4}{|c|}{ S. Em \pm} & \multicolumn{4}{|c|}{ S. Em \pm} \\
\hline $\mathbf{L}$ & \multicolumn{4}{|c|}{1.91} & \multicolumn{4}{|c|}{1.88} & \multicolumn{4}{|c|}{0.91} \\
\hline I & \multicolumn{4}{|c|}{1.83} & \multicolumn{4}{|c|}{1.81} & \multicolumn{4}{|c|}{0.93} \\
\hline L x I & \multicolumn{4}{|c|}{3.18} & \multicolumn{4}{|c|}{3.14} & \multicolumn{4}{|c|}{1.61} \\
\hline
\end{tabular}

Main plot: Land configuration $(\mathbf{L})$

$\mathbf{L}_{1}$ : Broad bed and furrow

$\mathbf{L}_{2}$ : Corrugated furrow (shallow)

$\mathbf{L}_{3}$ : Ridge and furrow

SV: Source of variation

\section{Sub Plot: Per cent depletion of available soil moisture (I)}

$\mathbf{I}_{\mathbf{1}}$ : Irrigation once in 10 days

I2: Irrigation at $40 \%$ depletion

$\mathbf{I}_{\mathbf{3}}$ : Irrigation at $50 \%$ depletion

I4: Irrigation at $60 \%$ depletion 
Table.5 Grain yield (q ha-1) of maize as influenced by land configuration and irrigation levels

\begin{tabular}{|c|c|c|c|c|c|c|c|c|c|c|c|c|}
\hline \multirow{2}{*}{ I/L } & \multicolumn{4}{|c|}{2015} & \multicolumn{4}{|c|}{2016} & \multicolumn{4}{|c|}{ Pooled } \\
\hline & $\mathbf{L}_{1}$ & $\mathbf{L}_{2}$ & $\mathbf{L}_{3}$ & Mean & $\mathbf{L}_{1}$ & $\mathbf{L}_{2}$ & $\mathbf{L}_{3}$ & Mean & $\mathbf{L}_{1}$ & $\mathbf{L}_{2}$ & $\mathbf{L}_{3}$ & Mean \\
\hline $\mathbf{I}_{1}$ & $64.6^{b c}$ & $69.2^{\mathrm{ab}}$ & $70.9^{\mathrm{ab}}$ & $68.2^{\mathrm{ab}}$ & $57.3^{\mathrm{cd}}$ & $62.2^{\mathrm{bc}}$ & $67.6^{\mathrm{ab}}$ & $62.4^{b}$ & $60.9^{\mathrm{ef}}$ & $65.7^{\mathrm{b}-\mathrm{e}}$ & $69.3^{\mathrm{a}-\mathrm{d}}$ & $65.3^{\mathrm{ab}}$ \\
\hline $\mathbf{I}_{2}$ & $67.6^{\mathrm{b}}$ & $73.2^{\mathrm{ab}}$ & $73.9^{\mathrm{ab}}$ & $71.6^{a}$ & $62.2^{b c}$ & $69.0^{\mathrm{a}}$ & $71.7^{\mathrm{a}}$ & $67.6^{a}$ & $64.9^{\mathrm{b}-\mathrm{e}}$ & $71.1^{\mathrm{a}-\mathrm{c}}$ & $72.8^{\mathrm{ab}}$ & $69.6^{\mathrm{a}}$ \\
\hline$I_{3}$ & $65.4^{b c}$ & $78.3^{\mathrm{a}}$ & $72.6^{\mathrm{ab}}$ & $72.1^{a}$ & $59.2^{\mathrm{cd}}$ & $71.6^{\mathrm{a}}$ & $71.0^{\mathrm{a}}$ & $67.3^{a}$ & $62.3^{\mathrm{d}-\mathrm{f}}$ & $74.9^{\mathrm{a}}$ & $71.8^{\mathrm{a}-\mathrm{c}}$ & $69.7^{\mathrm{a}}$ \\
\hline $\mathbf{I}_{4}$ & $57.3^{\mathrm{c}}$ & $66.6^{b c}$ & $69.6^{\mathrm{ab}}$ & $64.5^{b}$ & $53.3^{\mathrm{d}}$ & $61.3^{\mathrm{bc}}$ & $67.6^{\mathrm{ab}}$ & $60.8^{b}$ & $55.3^{f}$ & $64.0^{\mathrm{c}-\mathrm{e}}$ & $68.6^{\mathrm{a}-\mathrm{e}}$ & $62.6^{b}$ \\
\hline Mean & $63.7^{b}$ & $71.8^{\mathrm{a}}$ & $71.7^{\mathrm{a}}$ & & $58.0^{b}$ & $66.0^{\mathrm{a}}$ & $69.5^{\mathrm{a}}$ & & $60.9^{b}$ & $68.9^{a}$ & $70.6^{a}$ & \\
\hline SV & \multicolumn{4}{|c|}{ S. Em \pm} & \multicolumn{4}{|c|}{ S. Em \pm} & \multicolumn{4}{|c|}{ S. Em \pm} \\
\hline $\mathbf{L}$ & \multicolumn{4}{|c|}{1.80} & \multicolumn{4}{|c|}{1.57} & \multicolumn{4}{|c|}{1.63} \\
\hline I & \multicolumn{4}{|c|}{1.74} & \multicolumn{4}{|c|}{1.21} & \multicolumn{4}{|c|}{1.42} \\
\hline L $\times$ I & \multicolumn{4}{|c|}{3.01} & \multicolumn{4}{|c|}{2.10} & \multicolumn{4}{|c|}{2.46} \\
\hline
\end{tabular}

Table.6 Harvest index (\%) of maize as influenced by land configuration and irrigation levels

\begin{tabular}{|c|c|c|c|c|c|c|c|c|c|c|c|c|}
\hline \multirow{2}{*}{$\mathbf{I} / \mathbf{L}$} & \multicolumn{4}{|c|}{2015} & \multicolumn{4}{|c|}{2016} & \multicolumn{4}{|c|}{ Pooled } \\
\hline & $\mathbf{L}_{1}$ & $\mathbf{L}_{2}$ & $\mathbf{L}_{3}$ & Mean & $\mathbf{L}_{1}$ & $\mathbf{L}_{2}$ & $\mathbf{L}_{3}$ & Mean & $\mathbf{L}_{1}$ & $\mathbf{L}_{2}$ & $\mathbf{L}_{3}$ & Mean \\
\hline$I_{1}$ & $44.0^{\mathrm{ab}}$ & $46.2^{\mathrm{a}}$ & $45.8^{\mathrm{a}}$ & $45.3^{a}$ & $43.0^{\mathrm{ab}}$ & $42.7^{\mathrm{ab}}$ & $45.4^{\mathrm{a}}$ & $43.7^{\mathrm{a}}$ & $43.5^{\mathrm{ab}}$ & $44.5^{\mathrm{a}}$ & $45.6^{\mathrm{a}}$ & $44.6^{a}$ \\
\hline$I_{2}$ & $44.9^{\mathrm{ab}}$ & $46.8^{\mathrm{a}}$ & $45.1^{\mathrm{ab}}$ & $45.6^{a}$ & $44.3^{\mathrm{a}}$ & $45.7^{\mathrm{a}}$ & $44.0^{\mathrm{a}}$ & $44.7^{a}$ & $44.6^{\mathrm{a}}$ & $46.3^{\mathrm{a}}$ & $44.6^{\mathrm{a}}$ & $45.2^{\mathrm{a}}$ \\
\hline $\mathbf{I}_{3}$ & $44.0^{\mathrm{ab}}$ & $47.4^{\mathrm{a}}$ & $43.7^{\mathrm{ab}}$ & $45.0^{a}$ & $42.0^{\mathrm{ab}}$ & $46.4^{\mathrm{a}}$ & $43.1^{\mathrm{ab}}$ & $43.8^{a}$ & $43.0^{\mathrm{ab}}$ & $46.9^{\mathrm{a}}$ & $43.4^{\mathrm{ab}}$ & $44.5^{a}$ \\
\hline $\mathbf{I}_{4}$ & $40.3^{b}$ & $45.6^{\mathrm{a}}$ & $45.7^{\mathrm{a}}$ & $43.9^{a}$ & $38.2^{\mathrm{b}}$ & $43.1^{\mathrm{ab}}$ & $44.9^{\mathrm{a}}$ & $42.0^{a}$ & $39.2^{b}$ & $44.3^{\mathrm{a}}$ & $45.3^{\mathrm{a}}$ & $43.0^{a}$ \\
\hline Mean & $43.3^{a}$ & $46.5^{\mathrm{a}}$ & $45.1^{\mathrm{a}}$ & & $41.8^{a}$ & $44.5^{\mathrm{a}}$ & $44.3^{a}$ & & $42.6^{a}$ & $45.5^{\mathrm{a}}$ & $44.7^{\mathrm{a}}$ & \\
\hline SV & \multicolumn{4}{|c|}{ S. Em \pm} & \multicolumn{4}{|c|}{ S. Em \pm} & \multicolumn{4}{|c|}{ S. Em \pm} \\
\hline $\mathbf{L}$ & \multicolumn{4}{|c|}{1.09} & \multicolumn{4}{|c|}{1.21} & \multicolumn{4}{|c|}{1.01} \\
\hline I & \multicolumn{4}{|c|}{0.87} & \multicolumn{4}{|c|}{0.93} & \multicolumn{4}{|c|}{0.81} \\
\hline L x I & \multicolumn{4}{|c|}{1.51} & \multicolumn{4}{|c|}{1.61} & \multicolumn{4}{|c|}{1.40} \\
\hline
\end{tabular}

Main plot: Land configuration $(L)$

$\mathbf{L}_{\mathbf{1}}$ : Broad bed and furrow

$\mathbf{L}_{2}$ : Corrugated furrow (shallow)

$\mathbf{L}_{\mathbf{3}}$ : Ridge and furrow

SV: Source of variation
Sub Plot: Per cent depletion of available soil moisture (I)

$\mathbf{I}_{\mathbf{1}}$ : Irrigation once in 10 days

$\mathbf{I}_{2}$ : Irrigation at $40 \%$ depletion

$\mathbf{I}_{\mathbf{3}}$ : Irrigation at $50 \%$ depletion

I : Irrigation at $60 \%$ depletion 
Tumbatre and Bhoite (2000) recorded significantly higher yield parameters with ridges and furrow method. Similarly, irrigation at 40 per cent DASM produced significantly higher cob weight (182.1 g) and was on par with irrigation at 50 per cent DASM (179.4 g), whereas, statistically lowest cob weight was noticed with irrigation at 60 per cent DASM (165.4 g). Higher cob weight with irrigation at 40 per cent DASM might be associated with higher plant height and leaf area led to better source to sink relationship as compared to higher moisture depletion. Ahmed et al., (2015) recorded higher yield parameters of maize with irrigation at shorter irrigation interval. The interaction effect of ridges and furrow method at $50(189.7 \mathrm{~g})$ and 40 per cent DASM (188.7 g) recorded significantly higher and on par cob weight over the other treatments. However, the next best treatment in order was corrugated furrow method at 40 per cent DASM (183.8 g), whereas, significantly lowest cob weight was recorded with $\mathrm{BBF}$ method at 60 per cent DASM (142.7 g).

The grain yield of maize was significantly influenced by different land configurations, irrigation levels and interaction effect (Table 5). Ridges and furrow method (70.6 $\left.\mathrm{q} \mathrm{ha}^{-1}\right)$ and corrugated furrow method $\left(68.9 \mathrm{q} \mathrm{ha}^{-1}\right)$ found on par with each other but produced significantly higher grain yield as compared to BBF method of planting $\left(60.9 \mathrm{q} \mathrm{ha}^{-1}\right)$. The higher grain yield with ridges and furrow and corrugated furrow method might be due to improved growth parameters led to better dry matter accumulation, nutrient uptake and yield attributes. These results are in conformity with the findings of Thumbare and Bhoite (2000). Irrigation at 50 (69.7 $\mathrm{q}$ ha ${ }^{1}$ ) and 40 per cent DASM (69.6 q ha $\left.{ }^{-1}\right)$ recorded significantly higher and on par grain yield with irrigation once in ten days $(65.3 \mathrm{q}$ $\left.\mathrm{ha}^{-1}\right)$, whereas, irrigation at 60 per cent DASM recorded significantly lowest grain yield (62.6 q ha $\left.^{-1}\right)$. Higher grain yield with higher available soil moisture was attributed to higher photosynthesis and translocation of assimilates towards sink led to improved cob weight (Ahmed et al., 2015 and Yazar et al., 2009). The interaction effect was significantly with respect to grain yield of maize. Corrugated furrow with irrigation at 50 DASM recorded significantly higher grain yield (74.9 $\mathrm{q} \mathrm{ha}^{-1}$ ) as compared to other treatment combinations. However, this treatment remained on par with ridges and furrow at $40\left(72.8 \mathrm{q} \mathrm{ha}^{-1}\right)$ and 50 per cent DASM (71.8 q ha $\left.{ }^{-1}\right)$, whereas the BBF method of planting at 60 per cent DASM produced significantly lowest grain yield (55.3 $\left.\mathrm{q} \mathrm{ha}^{-1}\right)$.

Harvest index of maize did not respond to land configurations and irrigation levels (Table 6). However, harvest index was ranged from 42.6 to 45.5 per cent between land configurations and from 43.0 to 45.2 per cent among irrigation levels. Among interaction effect of harvest index was significantly with corrugated furrow method at different irrigation levels (44.3 to $46.9 \%$ ) and was at par with ridges and furrow method at varied irrigation levels (43.4 to $45.6 \%$ ), whereas, BBF method of planting at 60 per cent DASM recorded significantly lowest harvest index (39.2\%). Higher soil moisture availability and aeration throught the crop growth period might have improved the source to sink relationship and produced higher grain yield.

On the basis of results obtained in present investigation, it may be concluded that corrugated furrow method with irrigation at 50 per cent depletion of available soil moisture and ridges and furrow method with irrigation at 40 per cent depletion of available soil moisture significantly enhanced the growth and yield characteristics of maize over the rest of treatment combinations. Broad bed method of planting with irrigation at 60 per 
cent DASM recorded significantly lowest grain yield of maize.

\section{References}

Abdullah, K., Hayrettin Kușcu, Mehmet, O. and Gamze, B. 2015. The effect of different irrigation water levels on grain yield, yield components and some quality parameters of silage maize ( $\mathrm{Zea}$ mays indentata Sturt.) in Marmara Region of Turkey. Bot. Horti. Agrobo, 43(1): 138-145.

Ahmed, A., Alfalahi, H.M., Karem Al-Abodi, Bassam, K., Abdul Jabbar, Amer, M.M. and Khiadher, A.S. 2015. Scheduling irrigation as a water saving practice for corn (Zea mays L.) production in Iraq. Int. J. Appl. Agril. Sci., 1(3): 55-59.

Anonymous. 2015a. Vision 2050, Indian Institute of Maize Research, Indian Council of Agricultural Research, New Delhi. www.iimr.res.in. pp. 1-8.

Deshmukh, S.P., Jayesh, V. and Patel, A.M. 2016. A short review of land configuration to improve the plant growth, development and yield of cereals. Int. J. Interdisciplinary Res. and Innovations, 4(3): 1-4.

Fusheng, L., Jihua, L., Shaozhong, K. and Jianhua, Z., 2007, Benefits of alternate partial root-zone irrigation on growth, water and nitrogen use efficiencies modified by fertilization and soil water status in maize. Pl. and Soil, 295: 279291.

Girijesh, G.K., Kumaraswamy, A.S., Dinesh kumar, M., Nataraju, S.P., Vageesh, T.S. and Rajashekarappa, K.S. 2011. Response of maize (Zea mays L.) to constraint irrigation under late sown conditions. Karnataka J. Agric. Sci., 24(5): 633-635.

Kang, S., Liang, Z., Pan, Y., Shi, P. and Zhang, J. 2000. Alternate furrow irrigation for maize production in an arid area. Agril. Water Mgt., 45: 267274.

Michael, A.M. 2009. Irrigation Theory and Practice, Vikas Publishing House Pvt., p. 302.

Patil, S.L. and Sheelavantar, M.N. 2001. Effect of in situ moisture conservation practices and integrated nutrient management on nutrient availability and grain yield of rabi sorghum (Sorghum bicolor L.) in the vertisols of semi-arid tropics of south India. Ind. J. Agric. Sci., 71(4): 229-223.

Silungwe, F.R., Mahoo, H.F. and Kashaigili, J.J. 2010. Evaluation of water productivity for maize under drip irrigation. Second Ruforum Biennial Meeting 20-24 September 2010, Entebbe, Uganda: 725-728.

Tumbare, A.D. and Bhoite, S.U. 2000. Effect of moisture conservation techniques on growth and yield of pearl millet-gram sequence in watershed. Ind. J. Dry land Agric. Res. and Dev., 15(2): 94-95.

Yazar, A., Gokcel, F. and Sezen, M.S. 2009. Corn yield response to partial root zone drying and deficit irrigation strategies. Pl. Soil Environ., 55(11): 494-503.

\section{How to cite this article:}

Hanamant M. Halli and Angadi, S.S. 2017. Response of Land Configuration and Deficit Irrigation on Growth and Yield Attributes of Maize (Zea mays L). Int.J.Curr.Microbiol.App.Sci. 6(5): 52-60. doi: http://dx.doi.org/10.20546/ijcmas.2017.605.007 